\title{
Review of: "Probing atom dynamics of excited Co-Mo- S nanocrystals in 3D"
}

Ricardo Silva

Potential competing interests: The author(s) declared that no potential competing interests exist.

The article reports the magnitude of contrast in TEM images for the visualization of Co-Mo-S nanocrystals in 3D. Theoretical analysis using the analytical methodology with exit wave functions, the electrostatic potential and the atomic number of atoms that make up the nanocrystal, simulates crystalline growth through the analysis of time-resolved in-line holograms. Co-Mo-S nanocrystal stoichiometry is also analyzed based on theoretical exit wave. This theoretical methodology reproduces with excellent quality the morphological obtained by TEM images of Co-Mo-S nanocrystals The analytical methodology with exit wave functions is an excellent theoretical tool to collaborate with experimental data obtained by transmission electron microscopy images and with possible application to other microscopy techniques. 